\title{
Pentaquark and hybrid states
}

\author{
Elena Santopinto ${ }^{1, *}$ \\ ${ }^{1}$ INFN Sezione di Genova, via Dodecaneso 33, 16164 Genova, Italy
}

\begin{abstract}
We resume our results on the heavy quarkonium hybrids [19], and on the recently observed pentaquark states [16-18]. The latter have been investigated in three different scenarios: as compact pentaquark states [20], as a quantum superposition of $D^{(*)} \Lambda_{c}, D^{(*)} \Sigma_{c}$ and $D^{(*)} \Sigma_{c}^{(*)}$ meson-baryon states [30], and as a quantum superposition of meson-baryon molecular states and compact five-quark states [31].
\end{abstract}

\section{Charmonia $c \bar{c}$ and hybrids $c \bar{c} g$}

A deeper understanding of the importance of gluon degrees of freedom in hadron spectra has been possible through lattice simulations [1-3] and various effective models [4-11]. Among them the quasiparticle approach has had many successes, in particular, in the context of heavy quarkonia, i.e. flavorless mesons whose constituents are a heavy quark and its own antiquark. The effective potential, which originate from excited gluon configurations, can be computed using lattice simulations, and afterwards used to construct hybrid heavy quarkonia. When one keeps the quark-antiquark sources static and takes their separation to zero one obtains the spectrum of the so-called glue-lumps which describe the gluon field bound to a static color octet source. By starting from the study of the glue-lump, as obtained from QCD in physical gauge, it is easy to study the $c \bar{c}$ - gluon system, i.e. the hybrids. In [19] using the nonrelativistic reduction of Coulomb gauge QCD we computed the mass spectrum of the low mass heavy quarkonia. In this work the gluon degrees of freedom are treated in the mean field approximation calibrated to the gluelump spectrum. We also discussed the role of the non-Abelian nature of the QCD Coulomb interaction in the ordering of the spinparity levels, reported in Fig. 1. This different order of the spin-parity levels consists in the fact that, as one can see from Fig. 1 the lightest hybrid supermultiplet is the one with $J^{P C}=1^{-+}$, in agreement with lattice predictions (reported in the same figure). In 2005 the BaBar collaboration reported the observation of broad structure in $\pi^{+} \pi^{-} J / \Psi$ mass spectrum with spin-parity $J^{P C}=1^{--}$[14]. The mass of this state, about $4260 \mathrm{MeV}$, is very close to the predicted mass of the lightest hybrid supermultiplet. Thus, a possible hybrid nature of this state cannot be excluded.

\footnotetext{
*e-mail: Elena.Santopinto@ge.infn.it
} 


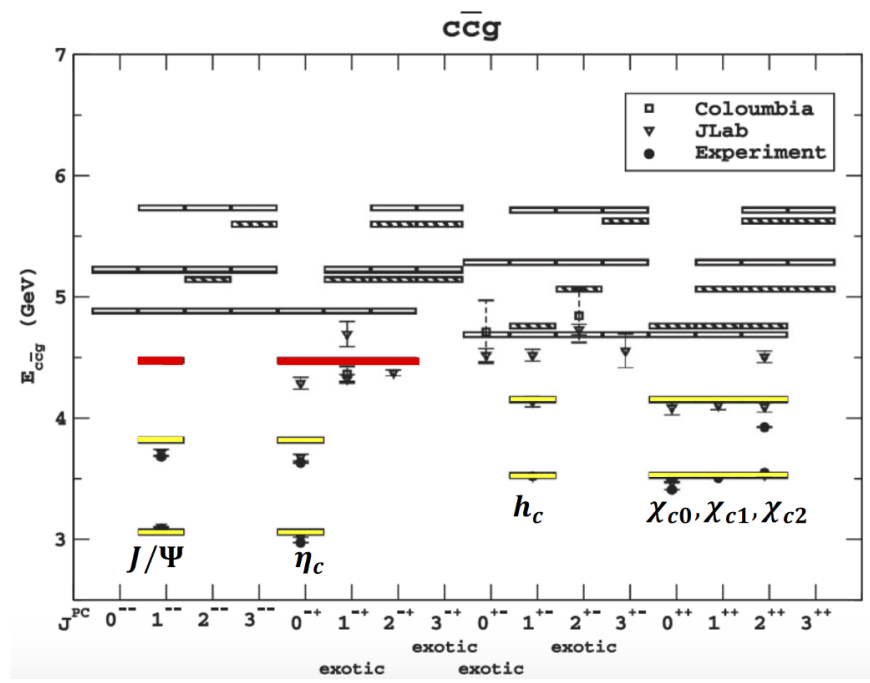

Figure 1. Charmonium (yellow boxes) and charmonium hybrid spectrum compared with data (where available) or lattice computations. The $c \bar{c}$ lightest states, $J / \Psi, \eta_{c}, h_{c}$ and $\chi_{c, J}$ are indicated explicitly. Red boxed are the lightest $c \bar{c} g$ hybrid supermultiplet. Open boxes are the $c \bar{c} g$ hybrids dominated by the $\mathrm{S}$-wave quarks, all other (dashed boxes) have the $Q \bar{Q}$ pair in the relative $\mathrm{P}$-wave orbital. Figure adapted from [19], APS copyright

\section{Pentaquark as compact states}

Recently, the LHCb collaboration reported the observation of two hidden-charm pentaquarks, $P_{c}^{+}(4380)$ and $P_{c}^{+}(4450)$, in $\Lambda_{b}^{0} \rightarrow J / \psi K^{-} p$ decay [16-18]. These masses of the two pentaquark states are $4380 \pm 8 \pm 28 \mathrm{MeV}$ and $4449.8 \pm 1.7 \pm 2.5 \mathrm{MeV}$, with corresponding widths of $205 \pm 18 \pm 86 \mathrm{MeV}$ and $39 \pm 5 \pm 19 \mathrm{MeV}$. The parities of these states are preferred to be opposite and one state has $J=3 / 2$ and the other $J=5 / 2 .\left(J_{P_{c}^{+}(4380)}^{P}, J_{P_{c}^{+}(4450)}^{P}\right)=\left(3 / 2^{-}, 5 / 2^{+}\right)$ gives the best fit solution, but also $\left(3 / 2^{+}, 5 / 2^{-}\right)$and $\left(5 / 2^{-}, 3 / 2^{+}\right)$are acceptable. In [20] we investigated the pentaquark states by means of a compact approach. Let $q=u, d$, s be a light quark and $c$ is the heavy charm quark. If we think pentaquark states as compact $q q q c \bar{c}$ states, we find that the allowed $S U_{f}$ (3) flavour representations to which the charmonium pentaquark states can belong are an octet and a decuplet [20]:

$$
[21]_{8},[3]_{10},
$$

where the notation $[f]_{d}=\left[f_{1}, \ldots, f_{n}\right]_{d}$, where $f_{i}$ denotes the number of boxes in the $i$-th row of the Young tableau, and $d$ is the dimension of the representation. With the following extension of the Gürsey-Radicati (GR) mass formula [29] (see [20] for the notation):

$$
M_{G R}=M_{0}+A S(S+1)+D Y+E\left[I(I+1)-\frac{1}{4} Y^{2}\right]+G C_{2}(S U(3))+F N_{C}
$$

and the assuming that the coefficients in the GR formula are the same for different quark systems one finds that lowest mass multiplet is is the $[21]_{8} S U_{f}(3)$ octet. This octet contains pentaquark ground states and for this reason, the pentaquark state observed by LHCb should belong to this octet. Actually, as displayed in Fig. 2, the charge state $P^{0+}(4377)$ has the same quantum numbers as the lightest resonance (charge, spin, parity) reported by the LHCb. The 
predicted mass of $P^{0+}(4377)$, according to the GR mass formula extension of Eq. 2 is $\mathrm{M}=$ $4377 \pm 49 \mathrm{MeV}$. We observe that the predicted mass is in agreement with the experimental mass, $4380 \pm 8 \pm 28 \mathrm{MeV}$ [16]. Observe also that the compact pentaquark approach predicts that the pentaquark $P^{0+}(4377)$ is a member of an isospin doublet, with hypercharge $Y=1$. In

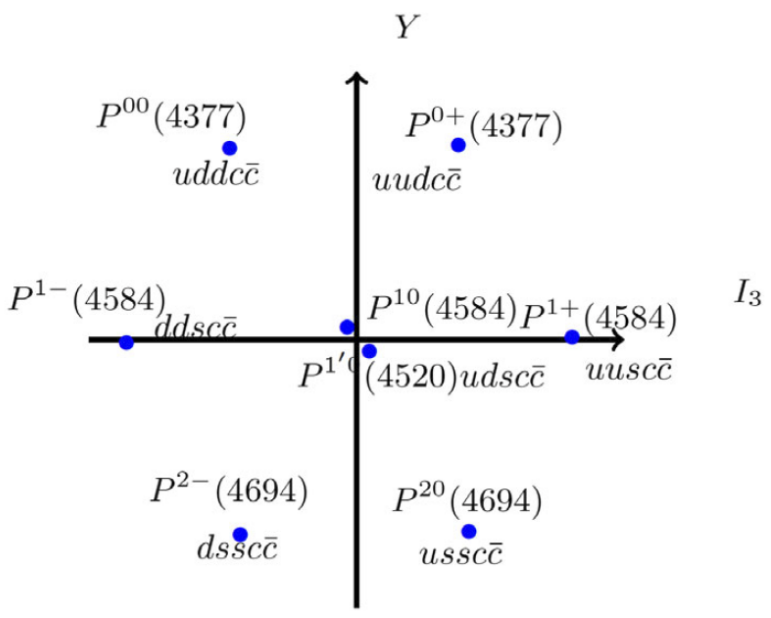

Figure 2. Octet of the charmonium pentaquark states. A pentaquark state is denoted with $P^{i j}(M)$, where $i=0,1,2$ is the number of strange quarks, $j=-, 0,+$ is the pentaquark's electric charge, and $M$ the predicted mass obtained with Eq. 2. Fig. taken from [20], APS copyright

calculating the decay widths of the predicted pentaquark states, reported in Fig. 2, we adopted the effective Lagrangian proposed by S. H. Kim et al. in [22]. Wang et al. [23] calculated the cross-section of the pentaquark states in $J / \Psi$ photoproduction and compared it with the available experimental data ([26-28]). Since the pentaquark states have been observed in $J / \Psi p$ channel, it should be produced also in $J / \Psi p$ photoproduction [23]. This provides a constraint for the $J / \Psi p$ coupling constant. In fact, in order to have consistency with the available photoproduction data the branching ratio upper limit for both the pentaquark states is $\mathcal{B}\left(P^{+} \rightarrow J / \Psi p\right) \leq 0.05$. From this constraint and the effective Lagrangian proposed by S. H. Kim in [22] we calculated the $J / \Psi p$ coupling constant, $\bar{g}_{p J / \Psi}=\frac{g_{1}}{2 M_{N}}$. Moreover, from our pentaquark mass predictions we were also able to calculate the partial decay widths for the pentaquark states with open strangeness. If we use the upper branching ratio limit extracted by Wang [23], $\mathcal{B}\left(P^{+} \rightarrow J / \Psi p\right)=0.05$, and the total width reported by $\mathrm{LHCb}$, $\Gamma_{t o t}=205 \mathrm{MeV}$, we obtain for $P_{c}$ (4380) partial decay width in $J / \psi p$ channel:

$$
\Gamma_{N J / \Psi}=\mathcal{B}\left(P^{+} \rightarrow J / \Psi p\right) \Gamma_{t o t}=10.25 \mathrm{MeV}
$$

The numerical results for the other channels are listed in Tab. 1.

\section{Pentaquarks as a meson-baryon molecule with coupled channels for $D^{(*)} \Lambda_{c}$ and $D^{(*)} \Sigma_{c}^{(*)}$}

Near the thresholds, resonances are expected to have an exotic structure, like the hadronic molecules. The observed pentaquarks are found to be just below the $D^{(*)} \Sigma_{c}\left(P_{c}(4380)\right)$ and 
Table 1. Partial decay widths for $\Lambda J / \Psi, \Sigma J / \Psi$ and $\Xi J / \Psi$ channels, calculated from the constraint that $J / \Psi p$ channel accounts for the $5 \%$ of the total pentaquark width, according to [23]

\begin{tabular}{ccc}
\hline \hline initial state & channel & $\begin{array}{c}\text { partial } \\
\text { width }(\mathrm{MeV})\end{array}$ \\
\hline$P^{1^{\prime} 0}$ & $\Lambda J / \Psi$ & 7.94 \\
$P^{1-}, P^{10}, P^{1+}$, & $\Sigma J / \Psi$ & 7.21 \\
$P^{2-}, P^{20}$, & $\Xi J / \Psi$ & 6.35 \\
\hline
\end{tabular}

$D^{(*)} \Sigma_{c}^{(*)}\left(P_{c}(4450)\right)$ thresholds. Moreover, as the $D^{(*)} \Lambda_{c}$ threshold is near the $D^{(*)} \Sigma_{c}^{(*)}$ threshold also the $D^{(*)} \Lambda_{c}$ channels are not irrelevant in the hidden-charm meson-baryon molecules. In [30] we considered the coupled channel systems of $D^{(*)} \Lambda_{c}, D^{(*)} \Sigma_{c}$ and $D^{(*)} \Sigma_{c}^{(*)}$, to predict the bound and the resonant states in the hidden- charm sector. In this work the binding interaction between the meson and the baryon is given by the One Meson Exchange Potential (OMEP). Fig. 3 displays the dependence of the pentaquark mass on the cut-off parameter $\Lambda$. If one fixes the cut-off parameter to reproduce the mass of the heaviest resonant state, $P_{c}(4450)$, one predicts of the lightest state a mass of about $4340 \mathrm{MeV}$, which is not much far from the experimental value, $4380 \pm 8 \pm 28 \mathrm{MeV}$.

\section{Pentaquarks as meson-baryon molecules coupled with five-quark states}

In [31] we study the pentaquark states as quantum superpositions of the meson-baryon channels and the five-quark compact core. The inclusion of the five-quark state is inspired by the recent work of Takeuchi et al. [32] and E. Santopinto [20]. In this work the coupling to the five-quark states is described as a short-range potential between the meson and the baryon, while the long-range force is given by the one-pion exchange potential. In this way it was possible to study the bound and resonant hidden-charm by solving the coupled channel Schrödinger equation for the isospin $I=\frac{1}{2}$ states with $J^{P}=\frac{1}{2}^{-}, \frac{3}{2}^{-}$, and $\frac{5}{2}^{-}$. Moreover, we extend also our investigation to hidden-bottom ground pentaquark states with quantum numbers $J^{P}=\frac{1}{2}^{-}, \frac{3}{2}^{-}$, and $\frac{5}{2}^{-}$. Given a set of quantum numbers, in the case in which more than one quantum state is allowed, the hadronic resonant states are unavoidably mixtures of these states. The two competing sets of channels to take into account in order to study the pentaquark states are the meson-baryon (MB) channels, which describe the dynamics at long distances and the five-quark (5q) channels, which describe the dynamics at short distances (1 fm or less). In Fig. 4 the two competitive channels are shown. The bound and resonant states are obtained by solving the coupled-channel Schrödinger equation for $\psi^{M B}$ which contains the one pion exchange potential, OPEP, $V^{\pi}(r)$, and the five-quark $5 q$ potential, $V^{5 q}(r)$. Fig. 5 shows the numerical results of the hidden-charm meson-baryon molecules: the bound and resonant state energies of the hidden-charm molecules are presented as a function of different values of the coupling constant $\frac{f}{f_{0}}$. The latter is a free parameter introduced to parametrise the coupling strength between the meson-baryon and the compact five-quark channels. The filled circles show the minimum coupling constant value needed for the formation of a resonant or bound state. For example, in the case of $J^{P}=\frac{5}{2}^{-}$, one resonance appears below the $\bar{D}^{*} \Sigma_{c}^{*}$ threshold when the coupling constant $\frac{f}{f_{0}}$ is larger than 25 . When the energy of a 
(i) $I\left(J^{P}\right)=1 / 2\left(3 / 2^{-}\right)$

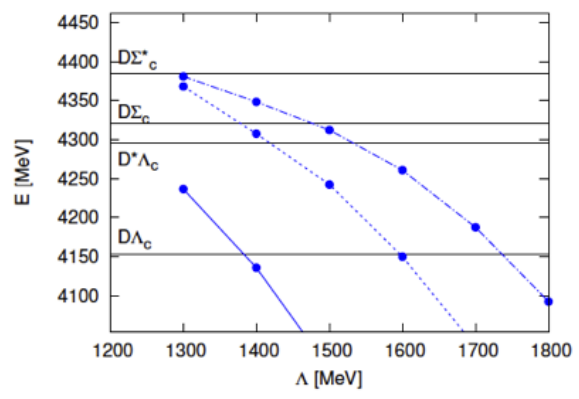

(iii) $I\left(J^{P}\right)=1 / 2\left(5 / 2^{-}\right)$

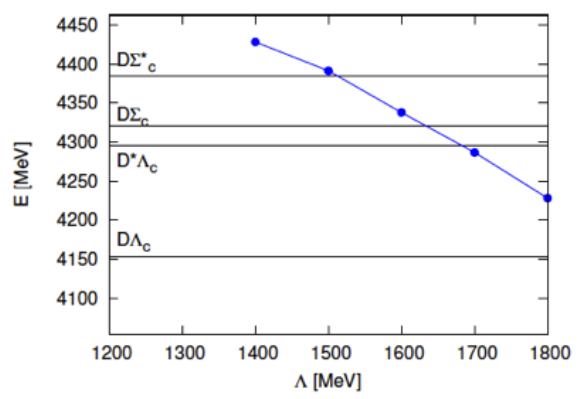

(ii) $I\left(J^{P}\right)=1 / 2\left(3 / 2^{+}\right)$

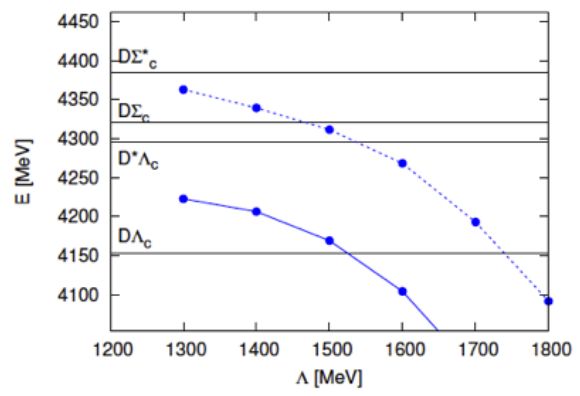

(iv) $I\left(J^{P}\right)=1 / 2\left(5 / 2^{+}\right)$

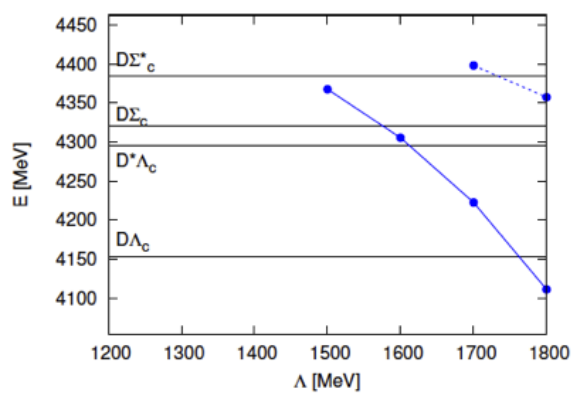

Figure 3. Cutoff $\Lambda$ dependence of the obtained energies $\mathrm{E}$ in for the pentaquark states with $J^{P}=$ $\frac{3}{2}^{-}, \frac{3}{2}^{+}, \frac{5}{2}^{-}$and $\frac{5}{2}^{-}$The solid, dashed and dot-dashed lines correspond to the obtained energy spectra of first, second and third states, respectively. The black horizontal lines show the meson-baryon thresholds.

Figure adapted from [30], APS copyright
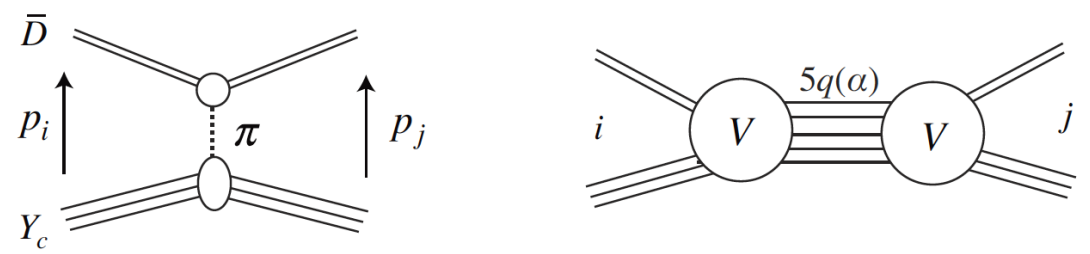

Figure 4. One pion exchange potential (left) and the effective interaction due to the coupling to the fivequark channel (right). The meson-baryon channels are represented by $D$ and $Y_{c}, i$ and $j$ are, respectively, the initial and the final channels, while a five-quark channel is denoted by $\alpha$. (APS copyright [31])

resonant state is lower than the lowest threshold, $\bar{D} \Lambda_{c}$, which is about $4150 \mathrm{MeV}$, the resonant state becomes a bound state. We observe that in these calculations all the meson-baryon components and the compact structure of the pentaquark states are taken into account at the same time, by solving the coupled channel Schrödinger equation of the system. The eigenvalues of the coupled channel Schrödinger equation, which are plotted in Fig. 5 as functions of the coupling constant $\frac{f}{f_{0}}$, are the resonant and the bound state energies. Thus, the resonant 

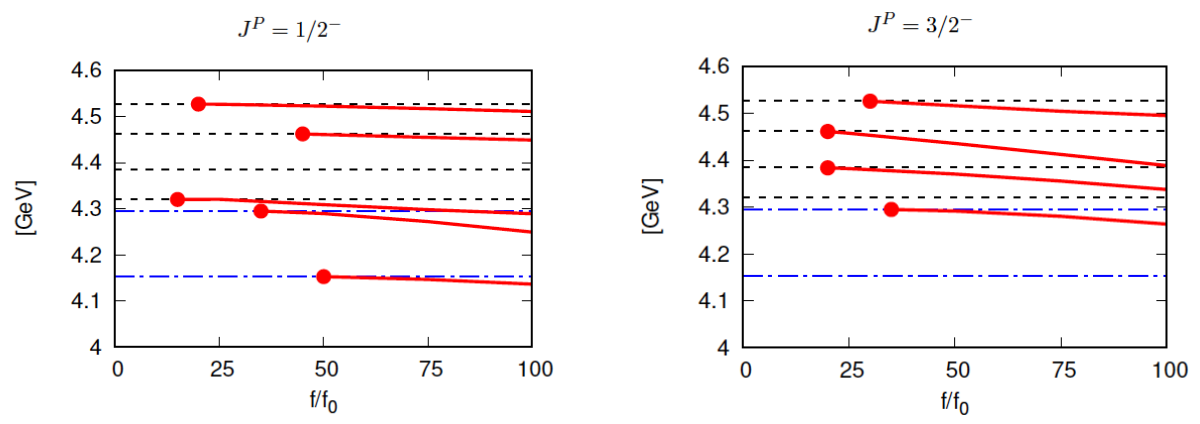

$J^{P}=5 / 2^{-}$

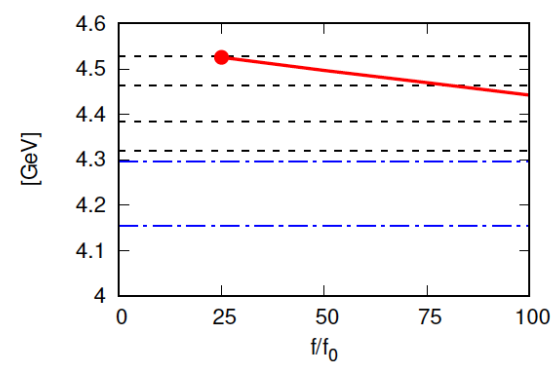

Figure 5. Bound and resonant state energies of the hidden-charm molecules (solid lines) with various coupling constants f. Dot-dashed lines are the $\bar{D} \Lambda_{c}$ and $\bar{D}^{*} \Lambda_{c}$ thresholds. Dashed lines are the $\bar{D} \Sigma_{c}, \bar{D} \Sigma_{c}^{*}, \bar{D}^{*} \Sigma_{c}$ and $\bar{D}^{*} \Sigma_{c}^{*}$ thresholds. The lowest threshold, $\bar{D} \Lambda_{c}$, is at $4150 \mathrm{MeV}$ and the state whose energy is lower than the threshold is a bound state (APS copyright [31])

or a bound states arise from the interactions between all the meson-baryon and the compact five-quark channels. We observe also that the fact that a state appears close to a particular meson-baryon threshold has not a particular meaning. In the hidden-charm sector, the one pion exchange potential (OPEP) is not enough strong to produce bound and resonant pentaquark states. In fact, as one can see from Fig. 5, no states are produced when the coupling constant $\frac{f}{f_{0}}$ is switched off, $\frac{f}{f_{0}}=0$.

Fig. 6 is similar as Fig. 5 but for it describes bottom sector. As one can see from Fig. 6 some bound states are produced without the necessity to add the five-quark potential, i.e., even when $\frac{f}{f_{0}}=0$. The only possibility to explain this behaviour is that the attractive interaction provided by OPEP is strong enough to bind the meson and baryon to produce stable hidden pentaquark structures. We notice also that the number of resonant and bound states increases when one switches on the $5 q$ potential.

\section{Conclusions}

The last study showed how the negative parity hidden-charm (hidden-bottom) pentaquark states are produced from the interplay between the S-wave configurations meson-baryon channels, and the compact five-quark core $u u d c \bar{c}(u u d b \bar{b})$. As a result we found that, unlike the hidden charm sector, the hidden bottom pentaquark states are produced even without introducing a coupling interaction between the compact five-quark configuration and the meson-baryon molecular configuration. This is a consequence of the fact that, in the hidden bottom sector, the kinetic energy of the meson-baryon system is suppressed with respect to 

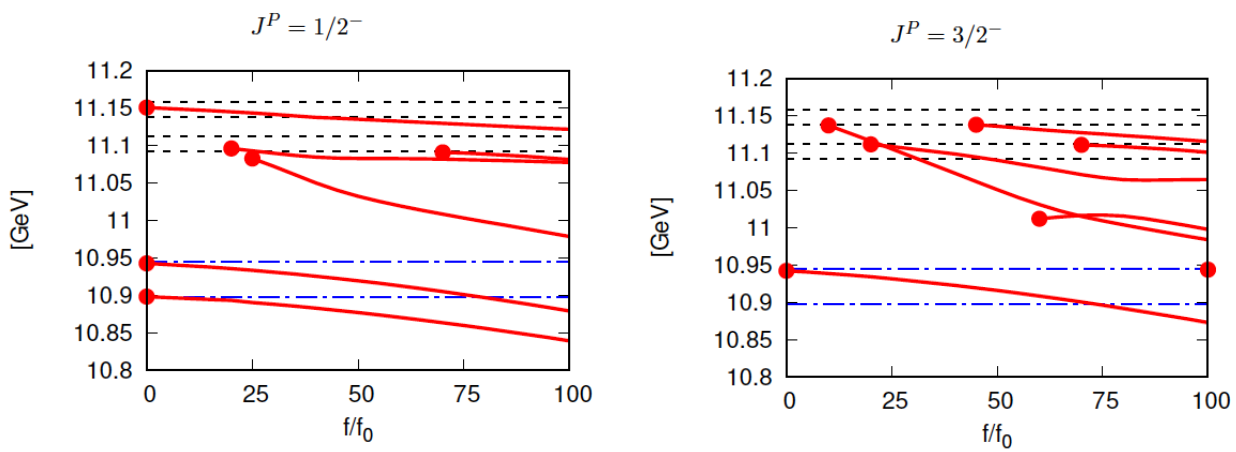

$J^{P}=5 / 2^{-}$

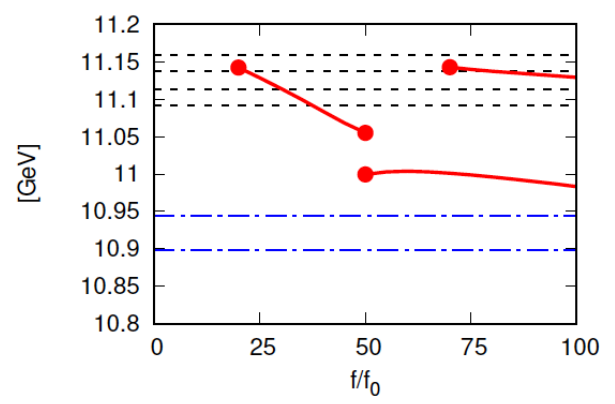

Figure 6. Bound and resonant state energies of the hidden-bottom molecules (solid lines) with various coupling constants f. Dot-dashed lines are the $\bar{B} \Lambda_{b}$ and $\bar{B}^{*} \Lambda_{b}$ thresholds. Dashed lines are the $\bar{B} \Sigma_{b}, \bar{B} \Sigma_{b}^{*}, \bar{B}^{*} \Sigma_{b}$ and $\bar{B}^{*} \Sigma_{b}^{*}$ thresholds. The lowest threshold, $\bar{B} \Lambda_{b}$, is at about $10900 \mathrm{MeV}$ and the state whose energy is lower than the threshold is a bound state (APS copyright [31])

the charm sector due to the higher reduced mass of the system. Moreover, in the bottom sector, the mixing effect are enhanced by the small mass splitting between the heavy mesons, $B$ and $B^{*}$, and between the heavy baryons $\Sigma_{b}$ and $\Sigma_{b}^{*}$. The higher stability of the hidden bottom pentaquark states than their hidden charm cousins should encourage experimentalists to search new resonant states in the hidden bottom sector. Finally we observe that another possibility to gain insight into the nature of the hidden charm and the hidden bottom pentaquark states consists of producing these states in a different reaction. In particular an observation pentaquark states in prompt production would indicate that the pentaquark has a compact nature, while on the contrary, a non-observation would not exclude the the possibility that they are molecular states.

\section{References}

[1] C. J. Morningstar and M. J. Peardon, Phys. Rev. D 60, 034509 (1999). arXiv:heplat/9901004

[2] M. Foster and C. Michael (UKQCD Collaboration), Phys. Rev. D 59, 094509 (1999). arXiv:hep-lat/9811010

[3] G. S. Bali and A. Pineda, Phys. Rev. D 69, 094001 (2004). arXiv:hep-ph/0310130

[4] D. Horn and J. Mandula, Phys. Rev. D 17, 898 (1978)

[5] N. Isgur and J. E. Paton, Phys. Rev. D 31, 2910 (1985) 
[6] Yu. A. Simonov, Nucl. Phys. B 592, 350 (2001). arXiv:hep-ph/0003114

[7] A. Szczepaniak, E. S. Swanson, C. R. Ji and S. R. Cotanch, Phys. Rev. Lett. 76, 2011 (1996)

[8] F. J. Llanes-Estrada and S. R. Cotanch, Phys. Lett. B 504, 15 (2001). arXiv:hep$\mathrm{ph} / 0008337$

[9] I. J. General, S. R. Cotanch and F. J. Llanes-Estrada, Eur. Phys. J. C 51, 347 (2007). arXiv:hep-ph/0609115

[10] F. Buisseret and C. Semay, Phys. Rev. D 74, 114018 (2006). arXiv:hep-ph/0610132

[11] F. Brau and C. Semay, Phys. Rev. D 70, 014017 (2004). arXiv:hep-ph/0412173

[12] E. Klempt and A. Zaitsev, Phys. Rept. 454, 1 (2007)

[13] S. K. Choi et al. (Belle Collaboration), Phys. Rev. Lett. 91, 262001 (2003)

[14] B. Aubert et al. (BABAR Collaboration) Phys. Rev. Lett. 95, 142001 (2005)

[15] A. Hosaka, T. Iijima, K. Miyabayashi, Y. Sakai, and S. Yasui, Prog. Theor. Exp. Phys. 2016, 062 C01 (2016). arXiv:1603.09229 [hep-ph]

[16] R. Aaij et al. (LHCb Collaboration), Phys. Rev. Lett. 115, 072001 (2015)

[17] R. Aaij et al. (LHCb Collaboration), Phys. Rev. Lett. 117, 082002 (2016)

[18] R. Aaij et al. (LHCb Collaboration), Phys. Rev. Lett. 117, 082003 (2016)

[19] P. Guo, A. P. Szczepaniak, G. Galata', A. Vassallo, and E. Santopinto Phys. Rev. D 78, 056003 (2008)

[20] E.Santopinto and A Giachino, Phys.Rev. D 96, 014014 (2017)

[21] M. Karliner and J. L. Rosner, Phys. Rev. Lett. 115, 122001 (2015). arXiv:1506.06386 [hep-ph]

[22] S. H. Kim, S. i. Nam, Y. Oh, and H.-Ch. Kim, Phys. Rev. D 84, 114023 (2011). arXiv:hep-ph/1110.6515

[23] Q. Wang, X. H. Liu, and Q. Zhao, Phys. Rev. D 92, 034022 (2015)

[24] J. J. Wu et al., Phys. Rev. Lett. 105, 232001 (2010)

[25] C. W. Xiao, J. Nieves and E. Oset, Phys. Rev. D 88, 056012 (2013). arXiv:1304.5368 [hep-ph]

[26] U. Camerini et al., Phys. Rev. Lett. 35, 483 (1975)

[27] R. L. Anderson. Report No. SLAC-PUB-1417

[28] B. Gittelman et al., Phys. Rev. Lett. 35, 1616 (1975)

[29] F. Gursey and L.A. Radicati, Phys. Rev. Lett. 13, 173 (1964)

[30] Y. Yamaguchi and E. Santopinto Phys. Rev. D 96, 014018 (2017)

[31] Y. Yamaguchi, A. Giachino, A. Hosaka, E. Santopinto, S. Takeuchi, M. Takizawa, Phys. Rev. D 96, 114031 (2017). hep-ph/1709.00819

[32] S. Takeuchi and M. Takizawa, Phys. Lett. B 764, 254 (2017). arXiv:hep-ph/160805475 